APA: Endrizzi, D. (2020). "Las dimensiones de la calidad democrática: un análisis metodológico de la propuesta de Leonardo Morlino.” En O. A. Agudelo Giraldo (Ed.), Derechos humanos, democracia y poder judicial (págs. 57-73). Bogotá: Editorial Universidad Católica de Colombia.

https://doi.org/10.14718/9789585133020.2020.3

Chicago: Endrizzi, Dimitri, "Las dimensiones de la calidad democrática: un análisis metodológico de la propuesta de Leonardo Morlino.” En Derechos humanos, democracia y poder judicial editado por Oscar Alexis Agudelo Giraldo, 57-73. Bogotá: Editorial Universidad Católica de Colombia.

doi: $10.14718 / 9789585133020.2020 .3$

\title{
LAS DIMENSIONES DE LA CALIDAD DEMOCRÁTICA: UN ANÁLISIS METODOLÓGICO DE LA PROPUESTA DE LEONARDO MORLINO
}

Dimitri Endrizzi

\section{Un cambio de objeto de estudio en la Ciencia Política}

A lo largo del desarrollo de las Ciencias Sociales, los acontecimientos históricos, en muchas ocasiones, han llevado la atención hacia un objeto de estudio específico. Si se quisiera encontrar el principal eje de interés de la Ciencia Política, y en particular de la Política Comparada, en las últimas décadas del siglo pasado, se deberían tomar en cuenta las democratizaciones. Estas conforman un fenómeno multifacético que interesó, en periodos relativamente cercanos, a todo el planeta.

Después de la Segunda Guerra Mundial, se dio lugar a lo que se conoce como el proceso de descolonización, prevalentemente en continentes como África y Asia. Este fue un fenómeno histórico-político, impulsado por Naciones Unidas, que se materializó a lo largo de diferentes etapas y que implicó la independencia de casi la totalidad de los países que habían sido víctimas de una colonización predominantemente europea (Martínez \& Moreno, 1999). 
Otros momentos relevantes que despertaron el interés académico, fueron los procesos de democratización en Europa Occidental y en América Latina -en particular los del Cono Sur-, alrededor, respectivamente, de las décadas de los setenta y ochenta. Por una parte, tras la muerte del General Franco, en noviembre de 1975, España emprendió un proceso de transición hacia la democracia, que culminaría en las elecciones del 15 de junio de 1977 y en el referendo que aprobó la Carta Magna, el 6 de diciembre de 1978 (Picarella, 2014; Rivas, 2014).

De igual manera, y más o menos contemporáneo al proceso español, fue el portugués. El 25 de abril de 1974, en Portugal hubo un levantamiento militar que puso fin a la dictadura de António de Oliveira Salazar y dio inicio al proceso de transición hacia la democracia (Soto, 2009).

Así mismo, en julio de 1974, Constantinos Karamanlís regresó del exilio a Grecia, después de varios meses de fuertes enfrentamientos entre militares golpistas, monarquía, militares demócratas y lo que hoy en día se llama sociedad civil. Karamanlís formó un gobierno democrático que, en noviembre del mismo año, convocó elecciones libres (Balios, 2018).

Por otra parte, entre los sesenta, setenta y ochenta, casi la totalidad de Latinoamérica se vio afectada por dictaduras militares, retomando gradualmente, hasta esa última década, el camino hacia la democracia (Serrano, 2010). Los casos emblemáticos, aunque no únicos, que todavía están presentes en la memoria colectiva, fueron los de Argentina y Chile.

Para terminar, y siguiendo las palabras de Huntington, la ola más reciente de los procesos de democratización que despertaron el interés académico tiene que ver con aquellos que se llevaron a cabo en Europa del Este tras la caída del muro de Berlín (1989).

Como se comentó anteriormente, los acontecimientos históricos han jugado un papel muy relevante en la determinación del objeto de estudio de la Ciencia Política. En el ámbito de los procesos de democratización, la Política Comparada ha producido investigaciones que son actualmente referentes importantes para cualquier académico que se acerque a la temática. Algunas de ellas son: Transition from an Authoritarian Rule, de Guillermo O'Donnell, Philippe C. Schmitter y Laurence Whitehead (1986); The Third Wave: Democratization in the Late Twentieth Century, de Samuel Huntington (1991); Elites and Democratic Consolidation in Latin America and Southern Europe, de John Higley y Richard 
Gunther (1992); los múltiples escritos de Valerie Bunce sobre los procesos de democratización en América Latina y, sobre todo, de Europa del Este; Problems of Democratic Transition and Consolidation: Southern Europe, South America, and Post-Communist Europe, de Juan Linz y Alfred Stepan (1996).

Cada vez más países cuentan con un sistema político que, de alguna forma, puede ser considerado como democrático, lo que implica la superación del umbral que les permite ser categorizados como tales. Es decir, los países cumplen con los requisitos básicos de una definición mínima de democracia, normalmente aceptada en el debate politológico. Entre dichos requisitos están: 1) el sufragio universal masculino y femenino; 2) las elecciones libres, competitivas, recurrentes y justas; 3) la existencia de más de un partido; 4) y las fuentes de información diversas y alternativas (Morlino, 2014).

Desde finales del siglo xx, las grandes olas de democratización han menguado y el principal foco de interés de la Ciencia Política se ha desplazado hacia otros objetos de estudio, entre los cuales está la calidad de las democracias ya consolidadas. Precisamente, Leonardo Morlino es uno de los académicos destacados que se ha dedicado al estudio de esta nueva temática.

A continuación se hará una reflexión, primero, sobre lo que se podría entender como "calidad de la democracia" y, segundo, sobre cómo este concepto se podría volver operativo a través de un proceso de operacionalización.

\section{De la definición del concepto}

¿Qué se entiende por calidad de una democracia? El discurso se hace complejo. Ya no se trata de determinar empíricamente si un país cumple o no con unos requisitos mínimos. Para aclarar, en ningún momento se quisiera afirmar que es una tarea fácil establecer un umbral pasado para que un país sea considerado como democrático. Esto requiere de algo que en las Ciencias Sociales es una mercancía rara: el consenso. De tal forma que este debe darse alrededor de una definición del concepto de democracia, así como de la altura a la cual se va a poner el listón que deberá ser superado. Sin embargo, medir la calidad de una democracia significa dar un paso más.

Dejando a un lado el debate sobre el consenso alrededor de una definición de democracia, es importante poner el foco sobre el concepto de calidad 
democrática. Pero, antes de explorarlo, es oportuno entender qué significa investigar este concepto. Según Morlino (2007), estudiar la calidad de una democracia implica incluir dos dimensiones específicas de la misma: una definición mínima y una máxima.

El autor, haciendo referencia al texto Democrazia e Definizioni, de Giovanni Sartori (1957), explica cómo el concepto de democracia lleva inevitablemente en sí mismo una dimensión empírica (definición mínima) y una dimensión ideal (definición máxima). Esta segunda dimensión juega un papel importante en la definición de la noción de calidad democrática y en su segmentación.

En cuanto a la dimensión empírica, esta es representada por unos requisitos mínimos necesarios, cuyo incumplimiento elimina la posibilidad de clasificar el régimen político como democrático. Es decir, la definición mínima fija un umbral -común para la literatura política-, conformado por los requisitos nombrados previamente: "sufragio universal, masculino y femenino; elecciones libres, competitivas, recurrentes, correctas; más de un partido; diferentes y alternativas fuentes de información" (Morlino, 2007, p. 5).

En ese orden de ideas, estudiar la calidad desde una perspectiva meramente empírica significaría, en primer lugar, tomar en consideración solamente los países que cuentan con un régimen político que supere el umbral establecido. En segundo lugar, declararía la no inclusión de ningún elemento ideal en la investigación.

Sin embargo, Morlino aclara que Sartori abre otro camino, instando a que se comparen las características empíricas observables en la realidad con los elementos ideales y normativos que hacen parte del "deber ser" de una democracia. En palabras del autor, "buscando los cotejos empíricos a través de un escrupuloso y cuidadoso análisis de qué tanto los ideales y normas éticas han logrado convertirse en una realidad efectiva" (Morlino, 2007, p. 4). O sea, se debe determinar qué hace falta para que los principales objetivos de una democracia ideal, libre e igualitaria, se materialicen en el régimen político que es objeto de estudio (Morlino, 2003).

Asimismo, Morlino (2007) hace referencia a un concepto general de calidad que debería trasladarse a un objeto de estudio tan complejo como la democracia. $\mathrm{Al}$ respecto, dice que 
1) la calidad es definida por los aspectos del procedimiento fijados cuidadosamente por cada uno de los productos, es decir, está en el seguir procedimientos constructivos precisos y controlados en los tiempos y en los métodos; el cuidado está en los procedimientos; 2) la calidad consiste en el tener un producto que tenga ciertas características constructivas, esté hecho con ciertos materiales, tenga formas y funcionamiento definidos, junto a otros aspectos del producto precisados en detalle: se pone, entonces, atención en el contenido; 3) la calidad del producto o del servicio deriva indirectamente de la satisfacción expresada por el consumidor, también en el volver a solicitar el producto o el servicio, independientemente de cuáles sean los procedimientos y sin necesidad de considerar directa y explícitamente los contenidos del producto o servicio o los procedimientos usados para tener ese producto o servicio, sino confiando simplemente en el resultado (Morlino, 2007, p. 5).

Como en toda investigación científica que utilice algún elemento teórico, el primer paso para determinar empíricamente la calidad de una democracia es definir el concepto de la misma. Según Morlino (2007), una democracia de calidad, o una "buena democracia", es: "esa ordenación institucional estable que a través de instituciones y mecanismos que funcionan correctamente, realiza la libertad y la igualdad de los ciudadanos" (p. 5). En esta definición, las dimensiones del concepto de calidad se relacionan, respectivamente, con procedimientos, contenidos y resultados. Cada dimensión influye en la caracterización de la investigación empírica.

Morlino (2007) enseña que una buena democracia es un régimen respecto al cual los ciudadanos están satisfechos y, por lo tanto, tiene plena legitimación (calidad respecto al resultado). Para que esto sea posible, las instituciones de dicho régimen tienen que gozar del apoyo de la sociedad civil. De esta manera, pueden cumplir con su labor de materialización de los valores del régimen en cuestión (calidad respecto al contenido).

Siguiendo lo anterior, si las instituciones, las cuales deben dedicar sus esfuerzos a su consolidación y mantenimiento, no pueden cumplir con una materialización de los valores del régimen más allá de la superación de un umbral, se podría decir que estas hacen parte de un régimen democrático, pero no de una buena democracia.

Para finalizar, los ciudadanos deben contar con un mecanismo eficaz para controlar y evaluar la manera en la que los dos macrovalores (libertad e igualdad) 
de una buena democracia se materializan en la sociedad. Lo anterior, tomando en consideración si este proceso se da dentro de los límites impuestos por la normatividad vigente, si la aplicación es eficiente, si las inquietudes expresadas por la sociedad civil han sido tenidas en cuenta y de qué manera esto ha sido ejecutado por los representantes democráticamente elegidos, etc. (calidad respecto al procedimiento).

¿De qué manera se definen los conceptos? Hay diferentes caminos. Una herramienta ampliamente utilizada en el ámbito de la Ciencia Política ha sido el triángulo de Ogden y Richards (figura 1), adoptado y propuesto principalmente por la Escuela de Sartori (Morlino, 2014). Esta herramienta, conocida también como triángulo de significación o triángulo semiótico, surge en el campo de la lingüística.

En su obra, The Meaning of the Meaning, los autores Charles Kay Ogden e Ivor Armstrong Richards, establecen una relación entre los tres componentes del significado, es decir, entre los tres vértices del triángulo. En este orden de ideas, el vértice derecho del triángulo es identificado con cualquier objeto que se pueda observar en la realidad empírica y se le conoce como referente; asimismo, el vértice alto del triángulo genera algún pensamiento o referencia; finalmente, el vértice izquierdo del triángulo representa dicho pensamiento o referencia a través de un símbolo.

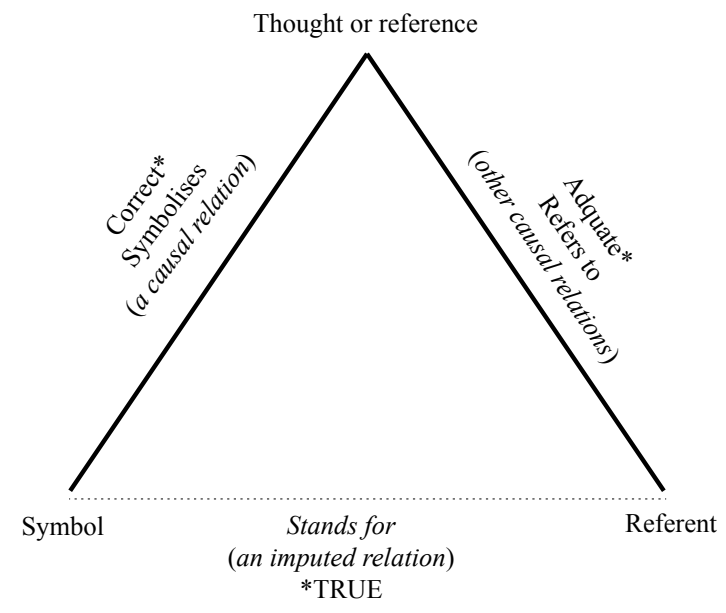

Figura 1. Triángulo de Odgen y Richards. 
Traducido al lenguaje de las Ciencias Sociales, un concepto es identificado a través de un término (vértice izquierdo), que presenta un significado (vértice alto), que a su vez permite observarlo en la realidad empírica, a través de un referente (vértice derecho). Siguiendo el ejemplo de Morlino (2014), un partido político (el término) es un conjunto organizado de personas que buscan, a través de medios legales, el control del aparato gubernativo (el significado), cuyo referente son todas las organizaciones observables en la realidad empírica, a través de la definición anteriormente presentada. En este sentido, un sindicato no entra en dicha categoría, porque sus funciones no corresponden a las expresadas en el significado. Por medio de la definición mencionada, solo se pueden observar los partidos políticos que operan en una realidad empírica objeto de estudio.

El triángulo de Ogden y Richards es importante para verificar la correcta definición de un concepto. En el caso de este texto, se puede aplicar de la siguiente manera: hay un término, representado por la expresión "buena democracia"; de igual forma, hay un significado, representado por la definición anteriormente reportada, en la que se expone que es "esa ordenación institucional estable que a través de instituciones y mecanismos que funcionan correctamente, realiza la libertad y la igualdad de los ciudadanos" (Morlino, 2007, p. 5); finalmente, hay un referente empírico, que son todos los países observables por medio de dicha definición. En otras palabras, la explicación que se le da al concepto, tal vez de forma no inmediata y directa, debe permitir encontrar algo en la realidad empírica.

La correcta definición de los conceptos es un paso fundamental para su sucesiva operacionalización. Si esto no ocurre, muy difícilmente se lograrán aplicar a la realidad empírica de forma efectiva. La operacionalización es entendida como los diferentes pasos para atribuir un contenido empírico a conceptos que no se pueden observar o percibir de forma directa en la realidad (Morlino, 2014).

Morlino distingue diferentes momentos del proceso de operacionalización de un concepto empírico: el primero es su definición; el segundo es conocido como segmentación, que implica la descomposición del concepto en sus dimensiones principales; el tercero, no abordado en el presente escrito, consta de la elaboración de indicadores, el mayor número posible, por cada dimensión. Hasta ahora, se ha dado el primer paso en este artículo. 


\section{La segmentación del concepto}

Las dimensiones de un concepto surgen de la definición que se le ha dado al mismo y están incluidas en una especie de "campo semántico". Al respecto, hay dos puntos que vale la pena destacar: uno de ellos, que se abordará más adelante, implica que dichas dimensiones deben mantenerse en el mismo nivel de abstracción.

El otro tiene que ver con que las dimensiones determinadas no pueden salirse del campo del concepto, de otra manera habría una situación que, siguiendo a Sartori, se podría definir como "estiramiento semántico". En esta, el significado de una dimensión específica desbordaría el campo del significado del concepto mismo y, en algunos aspectos, presentaría más características definitorias de las atribuidas a este.

Para simplificar un poco el discurso, sería como si se dividiera una manzana en diferentes partes y, al recomponerla, una parte de ella ya no encajara en la manzana original, porque es demasiado grande. Obviamente, en el ejemplo empleado hay una realidad física que lo impide. No obstante, cuando estamos hablando de conceptos empíricos, lo estamos haciendo sobre algo que no se puede observar ni percibir de forma directa en la realidad, sino que esto ocurre después de haberlos definido.

De acuerdo con Morlino (2007), la definición de buena democracia, anteriormente presentada, supone hablar de seis dimensiones (se verán a continuación) que pueden ser interpretadas como sus propiedades o sus cualidades. En palabras del autor, "se pasa de la calidad democrática más en general, como ha sido antes definida, a las calidades democráticas más específicas, como ahora se pueden precisar" (Morlino, 2007, p. 6). Vale la pena aclarar que las primeras tres dimensiones son de carácter exclusivamente procedimental, debido a que se relacionan solo indirectamente con los contenidos y se enfocan en las reglas del juego.

La primera dimensión es la denominada rule of law. Este concepto se relaciona con el respeto a la ley y con la garantía de un sistema legal que debe presentar unas características específicas, tales como: no retroactividad, publicidad, generalidad y estabilidad. A esto se suma el control civil de los militares y la independencia del poder judicial. Morlino (2007) propone una ulterior caracterización del concepto de rule of law, en relación con el de buena democracia, aludiendo a las siguientes propiedades: 
la aplicación erga omnes de un sistema legal, también supranacional, que garantiza derechos e igualdades de los ciudadanos; la consecuente ausencia, incluyendo el nivel local, de áreas dominadas por organizaciones criminales; la ausencia de corrupción en los aparatos políticos, administrativos, judiciales; la existencia de una burocracia civil, central y local, competente, eficiente y universalista en la aplicación de las leyes y responsable en caso de error; la existencia de fuerzas de policía eficientes y respetuosas de los derechos y de las libertades existentes y efectivamente garantizadas; el igual y fácil acceso de los ciudadanos a la justicia en caso de contencioso entre privados o bien entre privados e instituciones públicas; la razonable duración del proceso penal y del contencioso civil o administrativo; la completa independencia del juez o del jurado de cualquier influencia del poder político (p. 6).

La segunda dimensión es electoral accountability o responsabilidad electoral. Es lo que un elector o gobernado puede hacer valer frente a un elegido o gobernante, a través de un proceso periódico y cíclico. Haciendo referencia a una conceptualización de Andreas Schedler (1999), Morlino (2007) presenta los tres aspectos de la accountability: información, justificación y sanción o recompensa.

La información se relaciona con el actuar del político, o del organismo político en cuestión, y es un elemento necesario para evaluar la posible responsabilidad. La justificación se refiere a las razones provistas, por los entes descritos anteriormente, debido a su actuar. La sanción o recompensa se relaciona con el comportamiento del elector frente a una evaluación de la información disponible y la justificación.

En palabras simples, un elector decide si volver a votar por un candidato, teniendo en cuenta que la información disponible y la justificación otorgada por el mismo, en relación con su actuar, coincidan con sus expectativas respecto a la representación efectiva de sus intereses personales.

La tercera dimensión es interinstitucional accountability o responsabilidad interinstitucional. Es la "responsabilidad que hacen valer frente a los gobernantes otras instituciones o actores colectivos que tienen conocimientos y poder para evaluar el comportamiento de los gobernantes" (Morlino, 2007, p. 7). De acuerdo con O'Donnel (1999) y Schmitter (1999), citados por Morlino, se trata sustancialmente de un mecanismo de control de las actividades de gobierno, ejercido por otras instituciones, por ejemplo el parlamento, la magistratura constitucional (si 
está activa), los partidos políticos, los medios de comunicación, los sindicatos, las asociaciones empresariales, etc.

La cuarta dimensión es responsiveness o reciprocidad. Se relaciona con el resultado, "en el sentido de la capacidad de respuesta que encuentra la satisfacción de los ciudadanos y de la sociedad civil, más en general" (Morlino, 2007, p. 7). Siguiendo a Eulau y Karps (1977), Morlino presenta cuatro elementos que se vinculan entre sí, y a la luz de los cuales puede ser vista la reciprocidad. Entonces, refiere:

a las políticas en el centro de la atención pública, a los servicios para asegurar a los individuos y grupos que se representan, a la distribución de beneficios materiales a los propios representados a través de la administración pública o, de otro modo, a la donación de bienes simbólicos que crean, refuerzan o reproducen un sentido de confianza y apoyo de los representantes hacia los representados (Morlino, 2007, p. 7).

Las últimas dos dimensiones son las sustantivas de la buena democracia, en otras palabras, son los dos valores constitutivos de la misma: la libertad y la igualdad. Por una parte, la libertad se refiere a los derechos políticos y civiles. Por otra, la igualdad o solidaridad política, social y económica se traduce en los derechos sociales. En su nivel operativo, es algo que atañe a la disminución de la desigualdad presente y a la remoción de los principales obstáculos para alcanzar tal objetivo.

Es importante retomar lo que puntualiza Morlino (2007) respecto a la propuesta de Lijphart (1999) y de Altman y Pérez-Linan (2001) sobre la suma de dos dimensiones más, de carácter procedimental, al concepto de calidad democrática, que son la participación y la competición. Morlino (2007) expone:

La posición que sostengo aquí es que estas dos dimensiones, a pesar de ser muy importantes, sobre todo en una concepción liberal de la democracia, pueden ser condiciones importantes para el rule of law, la accountability, sobre todo la electoral, pero también para la reciprocidad y el respeto pleno por lo menos de los derechos de libertad. Es decir, se trata de aspectos que no conciernen directamente a la calidad democrática, pero sí a las condiciones de esta (p. 8).

La última frase de Morlino abre la puerta a una importante reflexión metodológica que se relaciona con la escala de abstracción propuesta por Sartori (2011). Este último autor, en un escrito que se ha vuelto un clásico de la Ciencia Política, Malformación de los conceptos en política comparada, plantea la problemática 
de los componentes verticales de un concepto, refiriéndose al "recorrido" de este a lo largo de la escala de abstracción.

Lo primero que Sartori destaca, es que una escala de abstracción se relaciona con conceptos empíricos, los cuales pueden ser reconducidos a una realidad observable o, con más precisión, a una realidad empírica. Es más, estos conceptos no solamente nacen del pensamiento teórico, sino que, en palabras del autor, son repetibles y observables.

Sartori (2011) dice que un investigador se enfrenta a una problemática doble. En primer lugar, porque tiene que determinar el nivel de abstracción de los conceptos empíricos. En segundo lugar, porque tiene que conocer las reglas que les permiten moverse, subir y descender, por la escala de abstracción. Para ello, el autor introduce una diferenciación entre denotación o extensión y otra entre connotación o intensión ${ }^{1}$ de un concepto.

Una definición normalmente compartida en la Ciencia Política, es que "la extensión de una palabra es la clase de cosas a la cual se aplica dicha palabra; la intensión de una palabra es el conjunto de propiedades que determinan las cosas a las cuales es aplicable esa palabra" (Sartori, 2011, p. 47). O sea, la connotación de un concepto refiere a sus características definitorias, mientras la extensión del mismo atañe a los objetos empíricos que se pueden observar a través de dichas características.

Sartori (2011) propone una primera regla básica para subir en la escala de abstracción, esto es, para aumentar el nivel de generalidad de un concepto, la cual consiste en que "para aumentar la extensión de un término se debe reducir su connotación” (p. 47). Como consecuencia, para disminuir la extensión de un concepto hay que aumentar su connotación. Este discurso solo es aparentemente complejo y, para entenderlo mejor, puede vérsele a través de un ejemplo.

El concepto de estudiante presenta una connotación muy baja y una extensión muy alta, lo que significa que ha sido definido de forma "pobre", en otras palabras, que las características que lo precisan son pocas. Por esta razón, puede abarcar una realidad empírica muy amplia. La Real Academia Española define el término estudiante como "persona que cursa estudios en un establecimiento de 
enseñanza" (RAE, 2019). Si se utilizara tal descripción como un lente para observar la realidad empírica, se podría abarcar a todos los estudiantes del mundo.

Pero, ¿qué sucede si se aumenta el nivel de definición del concepto? Es decir, ¿su connotación o intensión? Si se hablara de un(a) estudiante colombiano(a) de posgrado, el poder empírico de dicho concepto se reduciría. Los elementos (referentes) empíricos observables a través de su definición serían menores.

Los problemas suelen surgir en los extremos de la escala. Un concepto muy amplio, con un bajo nivel de connotación, tendrá un poder empírico muy alto, pero será tan general que no alcanzará a detectar las diferencias entre fenómenos. Por el contrario, un concepto demasiado detallado, con un nivel muy alto de connotación, logrará aplicarse a un número muy reducido de casos empíricos.

¿Cuál es la importancia de una escala de abstracción? Resumiendo un poco, cuando se baja a lo largo de ella, las dimensiones del concepto que se está determinando deben mantenerse en el mismo nivel de abstracción. De aquí lo que puntualiza Morlino, respecto a que la participación y la competición son condiciones importantes para dimensiones como rule of law, accountability, así como para la reciprocidad y el respeto pleno, por lo menos, de los derechos de libertad. No obstante, estas no pueden ser consideradas como dimensiones autónomas, sino que, algunas de ellas, podrían ser subdimensiones de la buena democracia.

El asunto no es marginal. Mantener las diferentes dimensiones de un concepto en el mismo nivel de abstracción es un paso fundamental, que tiene que ser respetado para que haya un correcto proceso de operacionalización de las mismas. Sartori ha insistido, a lo largo de toda su carrera académica, que no respetar esta regla puede llevar a una mala clasificación, la cual no permitirá que el concepto operativizado, en este caso el de la buena democracia, desprenda su potencial en términos de análisis empírico.

Para finalizar este texto, es fundamental comentar la conceptualización de Morlino a la luz de tres problemas, identificados por Tussel Collado y otros autores, que podrían afectar el proceso de análisis de la calidad de una democracia y que posiblemente estén presentes.

El primer problema es la interrelación entre dimensiones del concepto. Tussel Collado (2015), citando a Mazzuca (2007) y a Munck (2012), plantea la posibilidad de que las diferentes dimensiones de la noción de calidad democrática presenten relaciones de causa y efecto entre sí, por lo que no serían totalmente 
independientes. Morlino (2007) reconoce dicha situación y dice que existe una inevitable y continua interrelación entre las diferentes dimensiones de la buena democracia.

Ahora bien, ¿es algo que podría limitar el potencial analítico? Posiblemente no. La buena democracia y la democracia misma son conceptos con un altísimo nivel de complejidad. Ludwig von Bertalanffy (1976), con su teoría general de los sistemas, y los estudios sucesivos inspirados en ella, mostraron cómo las interrelaciones entre partes de un sistema son elementos fundacionales del mismo.

En este sentido, sin interrelaciones entre partes un sistema no se podría definir como tal. El problema no son entonces las conexiones de causa-efecto que intervienen entre las dimensiones del concepto de buena democracia propuesto por Morlino, sino una postura teórico-epistemológica que muestra las interrelaciones como una limitación.

El segundo problema que plantea Tussel Collado (2015), al igual que el anterior, puede relacionarse con la conceptualización de Morlino, y tiene que ver con el carácter ideal de algunas dimensiones de la noción de calidad democrática. Dicho autor adopta el discurso de Sartori, quien, de hecho, ni siquiera considera esto como un problema.

Para Sartori, incluir elementos ideales en la definición de conceptos y aterrizarlos a la realidad empírica es algo que enriquece la investigación. El paso obligatorio es la atribución de contenidos empíricos a los componentes ideales del concepto. El autor propone hacerlo, determinando el nivel de materialización de estos elementos en la realidad observada. Su discurso es muy sólido y abre espacios siempre novedosos en la investigación empírica. Lo ideal no presenta umbrales. Al respecto, y dando un paso más, se encuentra la siguiente inquietud: ¿Puede existir un concepto de calidad democrática sin un componente ideal?

Respecto al tercer problema, el de la caracterización personal y subjetiva, hay dimensiones del concepto de buena democracia que tienen que ver con el resultado, las cuales podrían irremediablemente estar vinculadas con percepciones. En particular, si se considera la responsabilidad electoral, ¿cómo evitar el elemento personal?

En lo que concierne a dicha dimensión, Morlino (2007) habla de tres aspectos: la información provista respecto al actuar político del elegido, la justificación por ello y el proceso de sanción-recompensa por parte del elector. Este último 
aspecto, en momentos electorales, es algo que no se relaciona con nada objetivo. Es un proceso que se refiere a las percepciones y valoraciones de un elector, en torno a la justificación de un gobernante sobre su actuar. Entonces, es válido preguntar, ¿cómo medir de forma objetiva una dimensión que pasa obligatoriamente por la percepción de un elector? Se puede, pero se estaría midiendo objetivamente algo que no lo es.

En este orden de ideas, son considerados como falsos problemas tanto la presencia de interrelaciones entre diferentes dimensiones del concepto, así como los elementos ideales en la investigación empírica y su carácter personal y subjetivo. El proceso de castigo-recompensa en una democracia es un elemento demasiado importante para ser ignorado solamente porque no es posible alcanzar una quimérica objetividad en el análisis.

\section{Conclusiones}

Al menguar las diferentes olas de democratizaciones, disminuyeron estos procesos como el principal objeto de estudio de la Ciencia Política. De esta manera, se ha ido dando paso, durante los últimos veinte años, a otras temáticas en la investigación politológica, como la calidad de las democracias ya consolidadas.

Leonardo Morlino ha sido uno de los principales académicos en el abordaje de este tema. El autor define una democracia de calidad como "esa ordenación institucional estable que, a través de instituciones y mecanismos que funcionan correctamente, realiza la libertad y la igualdad de los ciudadanos" (Morlino, 2007, p. 5).

En el proceso de operacionalización, el cual es necesario para atribuir un contenido empírico a conceptos abstractos que no se pueden observar ni percibir de forma directa en la realidad, el primer paso consiste en establecer de forma clara un término. Así, se hace posible que dicha noción pueda desarrollar su potencial analítico.

El segundo paso establecido por Morlino es segmentar el concepto, determinando sus principales dimensiones. El autor identifica seis: rule of law, accountability electoral, accountability interinstitucional, responsiveness, libertad e igualdad. De estas excluye dos dimensiones clásicas de la democracia, como son 
la participación y la competición, contempladas en los planteamientos de autores como Lijphart o Altman y Pérez-Linan.

Lo anterior, debido a un problema que se relaciona con la escala de abstracción de Sartori, aunque Morlino no lo expone específicamente en estos términos. Tanto la participación como la competición son vistas por el autor como características funcionales a la materialización de las demás dimensiones, pero no como dimensiones autónomas del concepto.

En lo que concierne a este documento, el proceso de segmentación del concepto llevado a cabo por Morlino no sufre de los principales problemas de los procesos de operacionalización, que son típicos en el ámbito del análisis politológico sobre la calidad de las democracias.

Es fundamental poder retomar las formas en las que los análisis de Morlino aportan a develar los falsos problemas relacionados a las dimensiones del concepto de calidad democrática. En primer lugar, la interrelación existente entre dimensiones, reconocida por el autor, es una característica intrínseca de dicho concepto. Esto solo podría originarse como "problema" en el ámbito de visiones teórico-epistemológicas que no logren contemplar dicha noción en toda su riqueza.

En segundo lugar, otros autores también han establecido como un "problema" el carácter ideal de algunos elementos del concepto de calidad democrática. Al respecto, vale la pena recordar que Sartori promueve el uso de elementos ideales, que se contrastan con la realidad empírica para observar en ella su materialización.

En tercer lugar, también se puede evidenciar como un falso problema el carácter subjetivo de algunas dimensiones, por ejemplo en lo que tiene que ver con la percepción del elector. Procesos como el castigo-recompensa de los votantes hacia los elegidos, están irremediablemente vinculados a percepciones y a falta de objetividad, lo cual no constituye razón suficiente para excluir dicha dimensión de un análisis que pretenda contemplar el concepto de calidad democrática. 


\section{Referencias}

Altman, D. \& Pérez-Liñán, A. (2001). Assessing the Quality of Democracy: Freedom, Competitiveness and Participation in 18 Latin American Countries. Democratization $9(2), 85-100$.

Balios, S. I. (2018). Grecia de la dictadura a la democracia (1974-1986): ¿Continuidad o ruptura? Historia Autónoma, (12), 225-242.

Bertalanffy, L. Von (1976). Teoría general de los sistemas: fundamentos, desarrollo, aplicaciones. México: Fondo de Cultura Económica.

Eulau, H. \& Karps, P. (1977). The Puzzle of Representation: Specifying Components of Responsiveness. Legislative Studies Quarterly, 2(3), 233-254.

Gómez Fernández, A. B. (2011). La llegada de la democracia al mediterráneo: las transiciones de Portugal, Grecia y España [en línea]. Historia Actual Online, (25), 7-18. Recuperado de https://dialnet.unirioja.es/servlet/articulo? codigo $=3670739$.

Huntington, S. (1991). The Third Wave: Democratization in the Late Twentieth Century. Norman: University of Oklahoma Press.

Lijphart, A. (1999). Patterns of Democracy: Government Forms and Performance in Thirty-six Countries. New Haven: Yale University Press.

Martínez, J. U. \& Moreno, J. (1999). Descolonización y Tercer Mundo. Cuadernos de Historia Contemporánea, (21), 147-160.

Morlino, L. (2003). Democrazie e democratizzazioni. Bologna: Il Mulino.

Morlino, L. (2007). Explicar la calidad democrática: ¿Qué tan relevantes son las tradiciones autoritarias? Revista de Ciencia Política, 27(2), 3-22.

Morlino, L. (2014). Introducción a la investigación comparada. Madrid: Alianza.

O’Donnell, G. (1999). Counterpoint: Selected Essays on Authoritarianism and Democratization. Notre Dame: University of Notre Dame Press.

Ogden, C. K. \& Richards, I. A. (1923). The Meaning of Meaning: A Study of the Influence of Language upon Thought and of the Science of Symbolism. Magdalene College: University of Cambridge.

Picarella, L. (2014). De la transición al zapaterismo: la evolución del sistema político español entre presidencialización y personalización. Bogotá: Planeta.

Real Academia Española, RAE (2019). Diccionario de la lengua española [en línea]; https://dle. rae.es/estudiante? $\mathrm{m}=$ form

Rivas, M. (2014). La transición española: la historia de un éxito colectivo. Aequitas, (4), 351-387. 
Salguero Lamillar, F. J. (2001). Teoría general de los signos y del significado. En A. Nepomuceno, J.F. Quesada \& F.J Salguero, Información: tratamiento y representación (pp. 41-58). Sevilla: Universidad de Sevilla.

Sartori, G. (1957). Democrazia e Definizioni. Bologna: Il Mulino.

Sartori, G. (2011). Cómo hacer ciencia política: lógica, método y lenguaje en las ciencias sociales. Madrid: Taurus.

Schedler, A. (1999). Conceptualizing Accountability. En A. Schedler, L.J. Diamond \& M. Plattner, (Eds.), The Self-restraining State: Power and Accountability in New Democracies (pp. 14-17). Boulder: Lynne Rienner.

Schmitter, P. (1999). The limits of horizontal accountability. En A. Schedler, L.J. Diamond \& M. Plattner (Eds.), The Self-restraining State: Power and Accountability in New Democracies (pp. 59-62). Boulder: Lynne Rienner.

Serrano, F. V. (2010). Estado, golpes de Estado y militarización en América Latina: una reflexión histórico-política. Nueva Época, 64, 175-193.

Soto, A. (2009). La transición a la democracia en el sur de Europa: la historia como instrumento para su comparación. Estudios Internacionales, 162, 7-30.

Tussel Collado, A. (2015). La calidad de la democracia y sus factores determinantes: un análisis comparado de 60 países. Politica y Sociedad, 52(1), 179-204. 
